\title{
A study of safety and tolerability of rotatory vestibular input for preschool children
}

\author{
This article was published in the following Dove Press journal: \\ Neuropsychiatric Disease and Treatment \\ 31 December 2014 \\ Number of times this article has been viewed
}

\author{
Wen-Ching Su' \\ Chin-Kai Lin' \\ Shih-Chung Chang ${ }^{2,3}$ \\ 'Program of Early Intervention, \\ Department of Early Childhood \\ Education, National Taichung \\ University of Education, Taichung, \\ Taiwan; ${ }^{2}$ Department of Physical \\ Medicine and Rehabilitation, Chung \\ Shan Medical University Hospital, \\ Taichung, Taiwan; ${ }^{3}$ School of Medicine, \\ Chung Shan Medical University, \\ Taichung, Taiwan
}

\begin{abstract}
The objectives of this study were to determine a safe rotatory vestibular stimulation input for preschool children and to study the effects of grade level and sex of preschool children during active, passive, clockwise, and counterclockwise rotation vestibular input. This study adopted purposive sampling with 120 children from three kindergarten levels (K1, K2, and K3) in Taiwan. The subjects ranged in age from 46 to 79 months of age (mean: 62.1 months; standard deviation $=9.60$ ). This study included testing with four types of vestibular rotations. The number, duration, and speed of rotations were recorded. The study found that the mean number of active rotations was 10.28; the mean duration of rotation was 24.17 seconds; and the mean speed was 2.29 seconds per rotation. The mean number of passive rotations was 23.04. The differences in number of rotations in clockwise, counterclockwise, active, and passive rotations were not statistically significant. Sex and grade level were not important related factors in the speed and time of active rotation. Different sexes, rotation methods (active, passive), and grades made significant differences in the number of rotations. The safety and tolerability of rotatory vestibular stimulation input data obtained in this study can provide useful reference data for therapists using sensory integration therapy.
\end{abstract}

Keywords: sensory integration, rotatory vestibular stimulation, tolerable input for rotatory vestibular stimulation

\section{Introduction}

Children with vestibular function disability may have underlying developmental delays. ${ }^{1-4}$ However, some of these children may have hyperresponsivity (a greater-thannormal response) or hyporesponsivity (a lower-than-normal response) to vestibular input, which affects their motor skill performance in daily life. ${ }^{5}$ If a child appears to have a sensory-seeking vestibular stimulus and lacks the ability to discriminate between stimuli, he may have a strong tolerability to vestibular sensation and thus might seek dangerous antigravitational activities to meet his needs. ${ }^{6}$

When occupational therapists perform sensory integration intervention, they usually have different interventions for individual clients' needs. Rotatory vestibular stimulation input is one of the most commonly used effective vestibular stimulation therapies, but there is still no reference value for rotatory vestibular input for occupational therapeutic intervention. Thus, it is important to establish a safe number of rotations and their duration as a reference for clinical application.

Ottenbacher ${ }^{7}$ reviewed the literature on vestibular stimulation, and indicated that vestibular stimulation activities included 1) rotatory stimulation, 2) linear or vertical acceleration, or 3 ) a combination of the two. Rotatory movement simulates the natural action the body uses when it acts as an axis for self-rotation.
Correspondence: Chin-Kai Lin Program of Early Intervention, Department of Early Childhood Education, National Taichung University of Education, 140 Min-Shen Road,

Taichung 40306, Taiwan

Tel +886 422183007

Fax +886422183400

Email linchinkai97@gmail.com
Neuropsychiatric Disease and Treatment 2015:II 4I-49 


\section{Functions of vestibular stimulation}

The vestibular system, which is part of the central nervous system, plays a key role in balance and spatial orientation. It is composed of the vestibular apparatus and the vestibular nerve. The vestibular receptor is located in the three semicircular canals, and the otolith is found in the inner ear. ${ }^{1}$ The vestibular apparatus, the major receptor in the human balance system, is located within the labyrinth in the inner ear in the temporal bone. The vestibular apparatus can be divided into the semicircular canals and the vestibule. The semicircular canals, horizontal, anterior, and posterior, are related to rotation and are perpendicular to each other. ${ }^{8,9}$ The flow of the endolymph in the semicircular canals touches the hair cells and detects the actions of rotation, acceleration, and deceleration. ${ }^{6}$ These dynamic messages are then sent to the vestibular nerve and the brain, and are integrated with other information to maintain balance. ${ }^{10}$ The vestibule is associated with balance, ${ }^{10}$ coordination, ${ }^{11}$ development of muscle tone, ${ }^{6,11,12}$ proximal joint stability, ${ }^{13}$ postural control,,${ }^{14,15}$ and plan of action. The vestibule is closely linked to the extraocular muscles, so it aids in development of visual motor integration. ${ }^{6,14}$ In addition, the vestibule can sense the spatial relationships of an individual within an environment and thus provides a sense of security ${ }^{16}$ and has a positive effect on an individual's psychological development.

\section{The application of assessments using rotatory vestibular stimulation}

The most common diagnostic assessment for vestibular function includes use of the postrotary nystagmus test ${ }^{17}$ and clinical observation. The postrotary nystagmus test is used to identify whether a child has a vestibular disability. ${ }^{18}$ During the test, the subject is seated on a rotation disk, with his or her head fixed in a 30-degree angle forward, to ensure that the semicircular canals are horizontal. ${ }^{19}$ Next, the subject is rotated for ten clockwise or counterclockwise rotations, at 2 seconds per rotation. The subject is asked to gaze forward with both eyes, and the examiner observes the degree of nystagmus produced by the rotations. ${ }^{2,20}$ We modified this test in our study investigating the safety and tolerability of rotatory vestibular input for children.

\section{The application of treatment using rotatory vestibular stimulation}

Sensory integration therapy provides stimulus input for the vestibular, proprioceptive, and tactile systems. ${ }^{13}$ Vestibular input is commonly used by occupational therapists in sensory integration therapy. Rotatory activities include the use of a rotating swing, rotating disk, jump circling, somersault, side rolling, or front rolling. Children with hyperresponsivity respond with gravitational insecurity or aversion to movement. In this situation, occupational therapists halt activities with the rotating stimulus until the vestibular sensory adaptation is normal. According to Lin's study, ${ }^{21}$ there were approximately $15 \%$ of children with hyporesponsivity issues. However, there were no data to indicate the number of children with hyporesponsivity for vestibular input. For most children with difficulty in sensory input and vestibular sensory-seeking, the therapist will provide vestibular input. If the rotatory vestibular stimulation input is excessive, it can cause an overload of stimulus to the brain, leading to an inability to concentrate, ${ }^{22}$ and causing the child to retreat and become defensive. It can also cause poor autonomic nervous system adaptation, such as unstable mood, dizziness, nausea, vomiting, paleness, hypotension, and postural imbalance. ${ }^{12}$

Occupational therapists observe these phenomena to determine whether there is an inappropriate reaction. However, it is not an easy job for a junior therapist to make an accurate observation of subtle change of sensory reaction. Thus, in sensory integration therapy, the number of rotations and controlling appropriate sensory input to produce an adaptive response are extremely important.

Since no studies have presented data on the safe number of rotations for children during vestibular testing, we designed a study to determine a safe, tolerable rotatory vestibular stimulation input for preschool children. In addition, we sought to investigate the effects of sex, grade, active and passive motion, and direction of rotation (clockwise, counterclockwise) on the number, time, and speed of rotations. Obtaining a reference value by evidence-based data ensures safe application of vestibular input in the intervention, and avoids poor adaptation.

\section{Materials and methods Participants}

This study adopted purposive sampling with children from three kindergartens (K1, K2, and K3) in Taiwan. Subjects were excluded if they had a confirmed diagnosis of developmental delay, because poor sensory perception and insufficient motor skills may affect vestibular input judgment and body balance adjustments, which would affect the test results. Children who were disabled were also excluded because most of these children have deficiencies in cognitive ability or motor skills. Those with vestibular dysfunction, such as paroxysmal positional vertigo or vestibular neuritis, Meniere's disease, migraine-associated vertigo, and childhood vertigo 
were also excluded from the study. Children with any of these conditions may become dizzy during rotation, which may be intolerable to them. Children with sensory deficits, such as myopia, hyperopia, strabismus, amblyopia, and sensorineural deafness, which may affect balance, were also excluded from the study. Children who had undergone prior sensory integration therapy were also excluded because their vestibular sensory systems have already been adjusted, which would affect the findings. We also excluded children who were in poor health on the day of the test; this included children with colds, injuries from falls, or unstable mood. Finally, children with a history of seizures were also excluded.

The final study group included 120 children, whose parents signed study consent forms. The subjects ranged in age from 46 to 79 months of age (mean: 62.1 months; standard deviation $[\mathrm{SD}]=9.60)$. There were 61 boys $(50.8 \%)$ and 59 girls (49.2\%); 49 were in K3 level classes (40.8\%), with a mean age of 72.02 months $(\mathrm{SD}=3.13)$. Forty children (33.3\%) were in K2 level classes, with a mean age of 59.83 months $(\mathrm{SD}=2.85)$. Thirty-one children were in level $\mathrm{K} 1$ classes $(25.8 \%)$, and the mean age was 49.35 months $(\mathrm{SD}=1.95)$. Table 1 provides the demographic characteristics of the population in this study. There was no statistically significant difference in grade by $\operatorname{sex}\left(\chi^{2}=0.91 ; P=0.64\right)$.

\section{Outcome measures}

This study included testing with four vestibular rotations: active clockwise rotation vestibular input, active counterclockwise rotation vestibular input, passive clockwise rotation vestibular input, and passive counterclockwise rotation vestibular input. The number, duration, and speed of rotations were recorded.

The postrotary nystagmus test, as described in the section "The application of assessments using rotatory vestibular stimulation", was adopted to measure vestibular input during passive rotation. The participants sat on a $60 \mathrm{~cm}$ diameter on the rotating disk. In active rotation, the participants stand on a $60 \mathrm{~cm}$ circle on the floor; the neck is fixed in a 30-degree

Table I Demographics of study participants

\begin{tabular}{|c|c|c|c|c|c|c|c|}
\hline \multirow[t]{3}{*}{ Level } & \multirow[t]{3}{*}{ Range } & \multirow[t]{3}{*}{$\mathbf{n}$} & \multirow[t]{3}{*}{$\%$} & \multicolumn{4}{|c|}{ Sex } \\
\hline & & & & \multicolumn{2}{|c|}{ Girls } & \multicolumn{2}{|c|}{ Boys } \\
\hline & & & & $\mathbf{n}$ & $\%$ & $\mathbf{n}$ & $\%$ \\
\hline K3 & $65-79$ & 49 & 40.8 & 22 & 44.90 & 27 & 55.10 \\
\hline $\mathrm{K} 2$ & $53-64$ & 40 & 33.3 & 22 & 55.00 & 18 & 45.00 \\
\hline $\mathrm{KI}$ & $46-53$ & 31 & 25.8 & 15 & 48.39 & 16 & 51.61 \\
\hline Total & $46-79$ & 120 & 100 & 59 & 49.2 & 61 & 50.8 \\
\hline
\end{tabular}

Abbreviation: $\mathrm{K}$, kindergarten. forward angle, while the body acts as the axis for selfrotation. The child controls the speed of active rotation, and the investigator calculates the speed of rotation by recording the duration and the number of rotations.

\section{Procedure}

Three thesis committees took training courses every year (which lasted at least three hours) from 2012 in order to meet the ethics requirements imposed by the Ministry of Science and Technology for grant application. The committees closely inspected all the study procedures to check that no harm was performed in a vulnerable population, examined if there were better ways to minimize discomfort and finally, checked that consent forms were signed by all parents. Then the investigator explained the purpose, procedure, and testing methods to the kindergarten teachers, who in turn explained the pertinent information to the parents when they arrived. Parents who consented to have their children receive the test according to the study protocol signed the study informed consent form. The parents and children were informed that this study was completely voluntary and refusal to participate would not impact the children's right in the kindergarten in any way.

Our study adopted a one-on-one design during the rotation test to avoid peer competition and other factors that might unduly influence the study outcome. To reduce any anxiety caused by unfamiliarity with the study staff, the child's teacher accompanied the child to the testing room. The test was conducted in the children's kindergarten but not in their usual classrooms. The testing environment was a brightly lit classroom that was free from noise or other distractions. Before the test, the investigator had simple interactions with the children to reduce any nervousness and to build trust. The investigator also had an opportunity to observe and assess the physical condition of the children and could use clinical observations and demographic data to exclude any of the children who did not meet the test criteria. During the pretest interactions, the investigator also helped prepare the children for the test.

The test was performed in the morning, before the usual morning snack time. If the children had already eaten, the test would be postponed for an hour to avoid gastrointestinal upset. Four tests were completed in 2 days, with active rotation on one day and passive rotation the next day. Clockwise rotation and counterclockwise rotation were performed on the same day, with a half-hour interval between clockwise and counterclockwise rotations. The test was conducted in the following order: active clockwise, active counterclockwise, passive clockwise, and passive counterclockwise rotations. The investigator used a metering device to record the number 
of rotations and a stopwatch to record the duration of rotations. Then, he observed and recorded the child's behavior after the rotations. The examination was halted if the child left the rotation disk during the test or complained of discomfort or of vertigo. It was also stopped if the children stopped the rotations on their own. The safety and tolerability of rotatory vestibular was defined in this study based on 1) the response of child, the child began to express discomfort, nausea, or dizziness or stopped on their own and 2) the phenomenon of autonomic nervous system, such as pale face, sweating, posture imbalance, and emotional instability.

During the test, if the child appeared to be in poor psychological or physiological health, for example, if he or she expressed discomfort, the test was stopped. The child could then rest, or another test time would be arranged. After the test, an appropriate amount of resistance exercise would be provided to relieve and adjust the results of vestibular stimulation; for example, squeezing a small ball, moving heavy objects, or pushing heavy objects. Children or their parents could ask to withdraw from the test at any time or refuse to participate in any test.

\section{Data analysis}

The Statistical Package for the Social Science (SPSS) (v 13.0; SPSS, Inc., Chicago, IL, USA) was used for data analysis. This study used statistical methods including descriptive statistics, dependent sample $t$-test, and mixed-design two-way analysis of variance (ANOVA). Scheffe tests were used for multiple post hoc comparisons when the variances were homogeneous. The level of statistical significance was set at 0.05 .

\section{Results}

\section{Differences in the number of rotations}

The results of the dependent sample $t$-test showed no significant differences in the number, duration, and speed of active clockwise and counterclockwise rotations. There also were no significant differences in the number of passive clockwise and counterclockwise rotations. Thus, this study used the mean number, time, and speed of clockwise and counterclockwise rotations to represent the number, time, and speed of rotations. The overall mean number of active rotations was $10.28(\mathrm{SD}=7.46)$, while the mean number of passive rotations was $23.04(\mathrm{SD}=31.4)$.

Our study adopted a two-way ANOVA approach with dependent samples. The grade times the mean number of rotations $(\mathrm{F})$, including the number of active and passive rotations, was $\mathrm{F}=4.63(P=0.01)$. The results indicated that the grade and the implementation had an interaction effect. Thus, simple main effect analysis was performed. Performance of simple main effect analysis included two steps. First, we used one-way ANOVA to analyze differences in the mean number of active rotations in children in different kindergarten levels, and differences of mean numbers of passive rotations for different levels. Second, we used the dependent sample $t$-test to analyze differences between the number of active and passive rotations among children in levels $\mathrm{K} 3, \mathrm{~K} 2$, and $\mathrm{K} 1$.

There was a significant difference between the mean number of active rotations in the different kindergarten levels. $\mathrm{F}=13.53(P=0.000)$ and the result of multiple post hoc comparisons indicated that the number of active rotations among those in level K3 was significantly greater than among those in levels $\mathrm{K} 2$ and $\mathrm{K} 1$, while there were no significant differences in levels $\mathrm{K} 2$ and $\mathrm{K} 1$ (Table 2). The mean number of passive rotations was significantly different in the three different levels; $\mathrm{F}=8.57(P=0.000)$ and the result of multiple post hoc comparisons indicated that the number of active rotations in level K3 was significantly greater than in levels $\mathrm{K} 2$ and $\mathrm{K} 1$, while there was no significant difference between levels $\mathrm{K} 2$ and $\mathrm{K} 1$. The number of active passive rotations was similar in the different levels (Table 2).

Table 2 Analysis to simple main effect of grad times implementation of rotation in mean rotation

\begin{tabular}{|c|c|c|c|c|c|c|c|c|c|}
\hline \multirow[t]{2}{*}{ Type } & \multirow[t]{2}{*}{ Level } & \multirow[t]{2}{*}{$\mathbf{M}$} & \multirow[t]{2}{*}{ SD } & \multicolumn{6}{|l|}{ ANOVA } \\
\hline & & & & Source of variation & SS & df & MS & $\mathbf{F}$ & Scheffe \\
\hline \multirow[t]{3}{*}{ Active rotations } & $\mathrm{K} 3$ & 14.05 & 9.79 & Between group & I,244.09 & 2 & 622.05 & $13.53 * * * *$ & $\mathrm{~K} 3>\mathrm{K} 2=\mathrm{K} \mathrm{I}$ \\
\hline & $\mathrm{K} 2$ & 8.50 & 3.35 & & & & & & \\
\hline & $\mathrm{KI}$ & 6.60 & 3.37 & Within group & $5,377.83$ & 117 & 45.96 & & \\
\hline \multirow[t]{3}{*}{ Passive rotations } & $\mathrm{K} 3$ & 36.00 & 42.02 & Between group & $\mid 4,997.79$ & 2 & $7,498.90$ & $8.57 * *$ & $\mathrm{~K} 3>\mathrm{K} 2=\mathrm{K} 1$ \\
\hline & $\mathrm{K} 2$ & 17.55 & 20.07 & & & & & & \\
\hline & $\mathrm{KI}$ & 9.65 & 7.94 & Within group & $102,341.00$ & 117 & 874.71 & & \\
\hline
\end{tabular}

Notes: $* * p<0.01 ; * * * P<0.001$.

Abbreviations: ANOVA, analysis of variance; df, degrees of freedom; K, kindergarten; SD, standard deviation; MS, mean square; M, mean rotation; SS, standard deviation. 
Analysis of dependent sample $t$-test results showed that the number of active and passive rotations of levels K3, K2, and $\mathrm{K} 1$ were significantly different. The $t$-test values for levels K3, K2, and K1 were $3.78(P=0.00), 2.89(P=0.00)$, and $2.29(P=0.03)$, respectively. Regardless of level, the number of passive rotations was significantly greater than was the number of active rotations. Effect sizes of differences of the number of active and passive rotations in levels $\mathrm{K} 3$, $\mathrm{K} 2$, and $\mathrm{K} 1$ were $2.24,2.70$, and 0.91 , respectively. Thus, the number of active and passive rotations was greatest in children in level K2.

The mean number of active rotations among level K3 students was 14.05 ( $\mathrm{SD}=9.79$ ); among level K2 students the number was $8.5(\mathrm{SD}=3.35)$ and, among level $\mathrm{K} 1$ students, the number was $6.60(\mathrm{SD}=3.37)$. The mean number of passive rotations among level $\mathrm{K} 3$ students was $36(\mathrm{SD}=42.02)$; among level K2 students this was 17.55 ( $\mathrm{SD}=20.07)$ and, among level K1 students, the number was 9.65 ( $\mathrm{SD}=7.94)$.

In regard to sex, two-way dependent sample testing did not show an interaction effect; therefore, a main effect test was performed. The mean number of active rotations for boys was 11.67 ( $\mathrm{SD}=9.16$ ), while the mean number of active rotations for girls was $8.83(\mathrm{SD}=4.81$ ). The mean number of passive rotations for boys was 28.84 ( $\mathrm{SD}=37.49$ ), and the mean number of passive rotations for girls was 17.04 $(\mathrm{SD}=22.29)$. After performing independent sample $t$-test analysis, the $t$-value of sex in the mean active rotations was $2.12(P=0.04)$. The number of active rotations among boys was significantly greater than among girls. The $t$-value of the mean number of passive rotations was $2.09(P=0.04)$; the number of passive rotations for boys was significantly greater than for girls (Table 3).

\section{Differences in the duration of rotation}

The duration of passive rotation was fixed at one rotation every 2 seconds; thus, only differences of the duration of active rotations were compared. After ANOVA, it was found that the mean duration of active rotations in the different kindergarten levels was $\mathrm{F}=13.64(P=0.00)$; thus, there were significant differences in the time of active rotations among different grade levels. Results of post hoc comparison suggested that the duration of active rotations among children in level K3 was significantly greater than among children in levels $\mathrm{K} 2$ and $\mathrm{K} 1$; there was no significant difference between children in levels $\mathrm{K} 2$ and $\mathrm{K} 1$. The overall duration of active clockwise rotations was 24.17 seconds $(\mathrm{SD}=20.05)$. The mean duration of active clockwise rotations among grade K3 students was 34.39 seconds ( $\mathrm{SD}=26.07$ ) versus $19.18 \mathrm{sec}$ onds ( $\mathrm{SD}=10.33$ ) among level K2 students; the mean duration of active clockwise rotation of level K1 students was 14.46 seconds ( $\mathrm{SD}=8.16$ ) (Table 4).

The mean time of active rotation for boys was 26.72 seconds ( $\mathrm{SD}=22.17$ ), compared to 21.54 seconds for girls $(\mathrm{SD}=17.38)$. After performing independent sample $t$-testing, the $t$-value of sex in mean time of active rotation was $1.42(P=0.16)$; thus, sex did not produce a significant difference in time of active rotations.

\section{Differences in the speed of rotation}

The overall speed of active clockwise rotations was 2.29 seconds per rotation $(\mathrm{SD}=0.66)$. The mean speed of active clockwise rotation of level $\mathrm{K} 3$ students was 2.38 seconds ( $\mathrm{SD}=0.64$ ); the mean speed of active clockwise rotation of level K2 students was 2.25 seconds ( $\mathrm{SD}=0.76$ ). The mean speed of active clockwise rotation of $\mathrm{K} 1$ students was 2.20 seconds ( $\mathrm{SD}=0.40$ ). After ANOVA comparison, it was found that the mean speed of active rotation in different grades was $\mathrm{F}=0.91(P=0.40)$; thus, level did not have a significant influence on the speed of active rotations.

The mean speed of active rotations for boys was 2.28 seconds ( $\mathrm{SD}=0.64$ ); the mean speed of active rotations for girls was 2.30 seconds ( $\mathrm{SD}=0.68$ ). After performing an independent sample $t$-test, the $t$-value of sex in mean time of active rotation was $-0.23(P=0.82)$; thus, there was no significant difference in the speed of active rotations due to sex.

Table 3 Differences by sex in mean number of active and passive rotations

\begin{tabular}{|c|c|c|c|c|c|c|c|c|}
\hline \multirow[t]{3}{*}{ Type of rotation } & \multicolumn{2}{|l|}{ Total } & \multicolumn{4}{|l|}{ Sex } & \multirow[t]{3}{*}{$t$} & \multirow[t]{3}{*}{ Comparison } \\
\hline & \multirow[b]{2}{*}{ M } & \multirow[b]{2}{*}{ SD } & \multicolumn{2}{|l|}{ Boys } & \multicolumn{2}{|l|}{ Girls } & & \\
\hline & & & $M$ & SD & $M$ & SD & & \\
\hline Active rotations & 10.28 & 7.46 & 11.67 & 9.16 & 8.83 & 4.81 & $2.12 *$ & Boys $>$ girls \\
\hline Passive rotations & 23.04 & 31.40 & 28.84 & 37.49 & 17.04 & 22.29 & $2.09 *$ & Boys $>$ girls \\
\hline
\end{tabular}

Note: $* P<0.05$

Abbreviations: $M$, mean; SD, standard deviation. 
Table 4 Variance analysis results for grades in duration of active rotations

\begin{tabular}{lllllllll}
\hline Level & Mean (seconds) & SD & ANOVA & & & \\
\cline { 4 - 8 } & & & Source of variation & SS & df & MS & F & Scheffe \\
\hline K3 & 34.39 & 26.07 & Between group & $9,043.78$ & 2 & $4,521.89$ & $13.64 * * *$ & $\mathrm{~K} 3>\mathrm{K} 2=\mathrm{K} 1$ \\
$\mathrm{~K} 2$ & 19.18 & 10.33 & & & & & \\
$\mathrm{KI}$ & 14.46 & 8.16 & Within group & $38,787.20$ & 117 & $331.5 \mathrm{I}$ & & \\
\hline
\end{tabular}

Note: $* * * P<0.001$.

Abbreviations: ANOVA, analysis of variance; df, degrees of freedom; K, kindergarten; SD, standard deviation; MS, mean square; SS, standard deviation.

\section{Discussion}

\section{The number of rotations}

\section{The effect of grade on the number of rotations}

The study results showed that the number of active and passive rotations was significantly greater among children in level K3 than among children in levels K2 and K1; there were no significant differences between children in grades $\mathrm{K} 2$ and K1. Cohen's effect value of differences in the number of rotations for children in levels K3 and K2 was 1.66; Cohen's effect value of difference for children in level K1 was 2.21; thus, the effect sizes were large.

There were three possible factors that may have affected the performance of level K3 students. The first was better mental arousal. During the test, level K3 children silently counted the number of rotations. Level K3 children had significantly more rotations. A number of studies have indicated that mental performance plays an important role in the rotation test, thus a higher mental arousal level would produce more nystagmus; $; 23,24$ this was also true in our study. A second factor might have been better motor skills among the children in level K3 than in the other two levels. According to the findings in a study conducted by Franjoine et $\mathrm{al}^{25}$ on the balance test for 2- to 13-year-olds, older children had better balance. In our study, the development of balance during the test was better among level $\mathrm{K} 3$ children than among those in levels K2 and $\mathrm{K} 1$. Active and passive rotations required the individual to be balanced; children in level $\mathrm{K} 3$ had a significantly larger number of rotations than did children in levels $\mathrm{K} 2$ and $\mathrm{K} 1$. A third element involved better strategy skills. Furman ${ }^{26}$ found that head position would affect the performance on the rotation test. In our study, we observed that level K3 children developed adaptive motor skills more easily during the test than did children in levels K2 and K1. An example of this was turning the head in the direction opposite to the rotation direction. Children in level K3 completed a greater number of rotations than did the other two groups.

\section{The difference of level in implementation}

The two-way simple main effect test showed that children in levels $\mathrm{K} 3, \mathrm{~K} 2$, and $\mathrm{K} 1$ performed a significantly greater number of passive, rather than active, rotations. One possible explanation was that different postures were required for the active and passive tests. The children stood for selfrotation in active rotation in this study. In contrast, being seated cross-legged for the passive rotation test, the number, speed, and time of rotations would be greatly affected by the strength of both hands and the child's balance. Children might not have been strong enough to push the rotation disk or may have pushed too hard, causing an imbalance and a fall from the rotation disk. During the procedure, different muscle strength and proprioceptive stimulus would affect the vestibular sensory input and the results of the study. Therefore, to reduce other sensory input in this study, for active rotation, the children stood for self-rotating; in contrast, for passive rotation testing, the children sat, and the investigator rotated the disk at a speed of one rotation per 2 seconds. The study results showed that the mean passive rotation was significantly greater than the mean active rotation. This was an unexpected result. The children being tested stood during self-rotation, which required more muscle strength of the lower limbs, as well as greater coordination and balance than was required for passive rotation. Thus, postural control could easily interfere and affect the number of rotations. In contrast, during passive rotation, the children were seated and passively received the rotations, which were controlled by the investigator. During the passive rotation test, the children had only to control their stability in a sitting position, and thus there was less interference from other vestibular input. This study found that the number of passive rotations was significantly greater than the number of active rotations.

\section{The effect of sex on the number of rotations}

The findings of this study showed that the number of active and passive rotations of boys was significantly greater than among the girls. Crowe et $\mathrm{al}^{27}$ adopted the Southern California Postrotary Nystagmus Test as a passive rotation test in a group of 4-year-olds. The results showed that the mean duration of periods of nystagmus after rotation for boys was longer than for girls. A study conducted by Ayres ${ }^{28}$ showed that girls had shorter periods of nystagmus after rotation. However, the 
focus of this study was the number of rotations rather than the timing of nystagmus. If the three outliers were removed in this study (the numbers of active rotation were 41,48 , and 50 , respectively, and passive rotations were 128,153 , and 205 , respectively), the $t$-value of sex, expressed as the mean number of active rotations, was $1.21(P=0.23)$. The $t$-value of the mean number of passive rotations was $1.84(P=0.69)$. It was found that there was no significant difference by sex in the number of active and passive rotations. The participants of these outliers like vestibular input particularly. Thus, the number of rotations was significantly different between boys and girls mainly because of these outliers. A study conducted by Casselbrant et $\mathrm{al}^{23}$ on the vestibulo-ocular reflex in an active rotation test for 3- to 9-year-old children reported no significant differences in performance according to sex. Studies conducted by Kimball ${ }^{2}$ and Punwar ${ }^{29}$ using the Southern California Postrotary Nystagmus Test showed no differences by sex during the test.

\section{Speed and duration of rotation}

The effect of grade on speed and duration of rotation

The speed of passive rotation was fixed at one rotation every 2 seconds; thus, only the variance analysis of the time and speed of active rotation was conducted. The results of ANOVA showed that the time of rotation of children in level $\mathrm{K} 3$ was significantly greater than for those in levels K2 and $\mathrm{K} 1$; differences in levels $\mathrm{K} 2$ and $\mathrm{K} 1$ were not significant. The possible reason was the longer time required for a greater number of rotations. Therefore, the duration of rotation required by children in level K3 was significantly greater than for those in levels K2 and K1.

The results of ANOVA showed that grade did not have a significant difference upon the speed of active rotation. One possible reason for this was the limitation of the selfrotating range during the test. The children could not step outward to maintain their body balance during rotation. To avoid falling, due to body imbalance or stepping outside the test area, the children slowed the speed of rotation and used a modest degree of self-rotation. The speed of rotation was 2.29 seconds per rotation. There was little difference with passive vestibular rotation at a speed of one rotation every 2 seconds. Children in levels K3, K2, and K1 used a strategy of adjusting the speed. Thus, there was no significant difference in the speed of active rotation based on grade.

\section{The effect of sex on the speed and duration of rotation}

The study results showed that sex did not have a significant effect on the duration and speed of active rotation. Participants with higher-speed rotation were prone to be imbalanced or stepped outside the rotation area. The investigator observed that the children would adjust the speed of rotation to prevent imbalance during the rotations. If participants were able to adjust the speed appropriately during the rotation, they would increase the speed of the rotations. Both boys and girls used a speed-adjustment strategy to maintain their balance. Therefore, sex did not have a significant difference in the duration and speed of rotation.

\section{The relationships with the rotation behavior}

This study recorded the degree of laughter and excitement as positive behavior after rotation. The degree of laughter and excitement was divided into extremely, very, fairly, slightly, and none, and was scored on a scale ranging from 5 (extremely) to 1 (none). This study found that the Pearson correlation coefficients of mean number of active and passive rotations and positive behavior were $0.3(P=0.00)$ and 0.6 $(P=0.00)$, respectively. The results indicated that a greater number of rotations resulted in more positive behaviors. However, the number of rotations did not have a significant correlation with negative behaviors. This finding indicated that a greater number of rotations would not result in more negative behaviors.

Negative behaviors were defined as unstable mood, anger, anxiety, dizziness, nausea, crying, yelling and screaming, refusing to continue the test, unstable posture, poor balance, and falling. One possible reason for development of negative behaviors was that the children were happy at the beginning of the test but, as the rotatory stimulation input gradually increased, they became unhappy or even felt aversion to the rotation. When negative behavior occurred, the participants would immediately stop the action of rotation. Thus, negative reactions of participants were not completely recorded. After analyzing the results, we found that the study did not cause adverse reactions among the participants. The behavior record after rotation was not a standardized assessment, and no reliability or validity tests were performed. The record of behavior served as a reference only. A future study might develop a standardized assessment of behavior after rotation, so that an in-depth analysis could be made.

\section{Suggestions}

According to the results of this study, the mean number of active rotations for level K3, K2, and K1 students was 14.05, 8.5 , and 6.60, respectively. The mean number of passive rotations for level K3, K2, and $\mathrm{K} 1$ students was 36, 17.55, and 9.65 , respectively. The mean number and duration of 
rotation can be used as reference data for one rotation. For example, the maximum number of passive rotation is no more than 36 when a therapist provides passive rotation for K3 students. Based on the child's grade and sex, one can establish a tolerable range, which can be helpful to vestibular input in therapy. The findings of this study showed that the SDs of mean duration of active and passive rotation were large, indicating that the individual differences were great in the participants. Therefore, when referring to the safe input suggested by this study, the sensory tolerability of individual clients has to be considered and adjusted when needed. For example, for children with hyperresponsivity of vestibular sensation or vestibular modulation disability, the input should be adjusted under the mean or only slightly. If the child has vestibular sensation-seeking or hyporesponsivity, the input should be adjusted above the mean, but no more than 1.96 SDs of the mean. The number and time of rotations can be adjusted according to the child's response after rotation.

We found that the child's head position, the size of the rotation area, degree of mental arousal, and sensory response all affect rotatory vestibular stimulation input. Therefore, it is suggested that a future study address the effects of the head position, the size of the rotation area, the degree of mental arousal, and the sensory response (such as under-discrimination to a stimulus, vestibular modulation disorder) on the number, time, and speed of vestibular rotation. Additional groups of children could also be investigated, such as children with developmental delay.

\section{Conclusion}

This study had two purposes: 1) to measure the safe rotatory vestibular stimulation input for preschool children, and 2 ) to study the effects of grade level and sex of preschool children during active, passive, clockwise, and counterclockwise rotation vestibular input. The study found that the mean number of active rotations was 10.28; the mean duration of rotation was 24.17 seconds; and the mean speed was 2.29 seconds per rotation. The mean number of passive rotations was 23.04. The differences in number of rotations in clockwise, counterclockwise, active, and passive rotations were not statistically significant. Sex and grade level were not important related factors in the speed and time of active rotation. Different sex, rotation methods (active, passive), and grades made significant differences in the number of rotations. The number of active and passive rotations of boys was significantly greater than was the case among girls. The mean number of active and passive rotations of children in level K3 was significantly greater than those in levels K2 and $\mathrm{K} 1$; there was no significant difference between children in levels $\mathrm{K} 2$ and $\mathrm{K} 1$. The safety and tolerability of rotatory vestibular stimulation input data obtained in this study can provide useful reference data for therapists using sensory integration therapy.

\section{Acknowledgments}

The authors would like to thank National Science Council of Taiwan (100-2410-H-142-005-2410-H-142-005) and Ministry of Science and Technology of Taiwan (101-2410H-142-005-MY3) for supporting this research.

\section{Disclosure}

The authors report no conflicts of interest in this work. No commercial party having a direct financial interest in the results of the research supporting this article has or will confer a benefit upon the authors or upon any organization with which the authors are associated.

\section{References}

1. Cronin GW. Pediatric vestibular disorders. Retrieved November. 2012; 15:1-8.

2. Kimball JG. Normative comparison of the Southern California postrotary nystagmus test: Los Angeles vs Syracuse data. Am J Occup Ther. 1981;35(1):21-25.

3. Suarez H, Angeli S, Suarez A, Rosales B, Carrera X, Alonso R. Balance sensory organization in children with profound hearing loss and cochlear implants. Int J Pediatr Otorhinolaryngol. 2007;71(4):629-637.

4. Weiss AH, Phillips JO. Congenital and compensated vestibular dysfunction in childhood: an overlooked entity. J Child Neurol. 2006;21(7):572-579.

5. White BP, Mulligan S, Merrill K, Wright J. An examination of the relationships between motor and process skills and scores on the sensory profile. Am J Occup Ther. 2007;61(2):154-160.

6. Kranowitz CS. The Out-Of-Sync Child: Recognizing and Coping with Sensory Integration Dysfunction. New York: A Skylight Press Book/A Perigee Book; 2005

7. Ottenbacher K. Developmental implications of clinically applied vestibular stimulation. Phys Ther. 1983;63(3):338-342.

8. Fridman GY, Della Santina CC. Progress toward development of a multichannel vestibular prosthesis for treatment of bilateral vestibular deficiency. Anat Rec (Hoboken). 2012;295(11):2010-2029.

9. Herdman SJ. Vestibular Rehabilitation. Philadephia, PA: F.A. Davis Company; 2007.

10. Kramer P, Hinojosa J. Frames of Reference for Pediatric Occupational Therapy. Philadelphia, PA: Lippincott Williams \& Wilkins; 2010.

11. Mulligan $\mathrm{S}$. Validity of the postrotary nystagmus test for measuring vestibular function. OTJR (Thorofare $N J$ ). 2011;32(2):97-103.

12. Lang E, Walsh RM. Vestibular function testing. Ir J Med Sci. 2010;179(2):173-178.

13. Bundy AC, Lane SJ, Murray EA. Sensory Integration: Theory and Practice. Philadelphia, PA: F.A. Davis Company; 2002.

14. Angeli S. Value of vestibular testing in young children with sensorineural hearing loss. Arch Otolaryngol Head Neck Surg. 2003;129(4):478-482.

15. Kandel ER, Schwartz JH, Jessell TM. Essentials of Neural Science and Behavior. Norwalk, CT: Appleton and Lange; 1995. 
16. Royeen CB. Incidence of hypoactive nystagmus among behaviorally disordered children. Occupational Therapy Journal of Research. 1984;4(31):237-240.

17. Cohen B. Erasmus Darwin's observations on rotation and vertigo. Hum Neurobiol. 1984;3(3):121-128.

18. Kramer LA, Crowe TK, Deitz JC. Effect of activity level on Southern California Postrotary Nystagmus Test scores. Occupational Therapy Journal of Research. 1988;8:345-355.

19. Mora R, Dellepiane M, Barettini L, Crippa B, Salami A. Electronystagmography in migraine equivalent syndrome. Otolaryngol Head Neck Surg. 2009;140(4):566-572.

20. Ayres, AJ. Sensory Integration and Praxis Tests. Los Angeles: Western Psychological Services; 1989.

21. Lin CK. Test of Sensory Integration Function: User's Manual. Taipei: Psychological Corporation; 2004.

22. Miller LJ, Anzalone ME, Lane SJ, Cermak SA, Osten ET. Concept evolution in sensory integration: a proposed nosology for diagnosis. Am J Occup Ther. 2007;61(2):135-140.
23. Casselbrant ML, Mandel EM, Sparto PJ, et al. Longitudinal posturography and rotational testing in children three to nine years of age: normative data. Otolaryngol Head Neck Surg. 2010;142(5):708-714.

24. Montgomery PC, Rodel DM. Effect of state on nystagmus duration in the Southern California Postrotary Nystagmus Test. Am J Occup Ther. 1982;36(3):177-182.

25. Franjoine MR, Darr N, Held SL, Kott K, Young BL. The performance of children developing typically on the pediatric balance scale. Pediatr Phys Ther. 2010;22(4):350-359.

26. Furman JM. Rotational testing: background, technique and interpretation. Handbook of Clinical Neurophysiology. 2010;9:141-149.

27. Crowe TK, Deitz JC, Siegner CB. Postrotatory nystagmus response of normal four-year-old children. Phys Occup Ther Pediatr. 1984;4(2): $19-28$

28. Ayres AJ. Southern California Postrotary Nystagmus Test Manual. Los Angeles, CA: Western Psychological Services; 1975.

29. Punwar A. Expanded normative data: Southern California Postrotary Nystagmus Test. Am J Occup Ther. 1982;36(3):183-187.
Neuropsychiatric Disease and Treatment

\section{Publish your work in this journal}

Neuropsychiatric Disease and Treatment is an international, peerreviewed journal of clinical therapeutics and pharmacology focusing on concise rapid reporting of clinical or pre-clinical studies on a range of neuropsychiatric and neurological disorders. This journal is indexed on PubMed Central, the 'PsycINFO' database and CAS,

\section{Dovepress}

and is the official journal of The International Neuropsychiatric Association (INA). The manuscript management system is completely online and includes a very quick and fair peer-review system, which is all easy to use. Visit http://www.dovepress.com/testimonials.php to read real quotes from published authors.

Submit your manuscript here: http://www.dovepress.com/neuropsychiatric-disease-and-treatment-journal 